\title{
DOES BUCKLING INSTABILITY OF THE PSEUDOPODIUM LIMIT HOW WELL AN AMOEBA CAN CLIMB?
}

\author{
SANDIP GHOSAL \\ DEPARTMENT OF MECHANICAL ENGINEERING \\ MCCORMICK SCHOOL OF ENGINEERING AND APPLIED SCIENCE \\ NORTHWESTERN UNIVERSITY \\ 2145 SHERIDAN ROAD, EVANSTON, IL 60208 \\ E-MAIL: S-GHOSAL@NORTHWESTERN.EDU \\ YOSHIO FUKUI \\ DEPARTMENT OF CELL AND MOLECULAR BIOLOGY \\ FEINBERG SCHOOL OF MEDICINE \\ NORTHWESTERN UNIVERSITY \\ 303 EAST CHICAGO AVENUE, CHICAGO, IL 60611 \\ E-MAIL: Y-FUKUI@NORTHWESTERN.EDU
}

\begin{abstract}
The maximum force that a crawling cell can exert on a substrate is a quantity of interest in cell biomechanics. One way of quantifying this force is to allow the cell to crawl against a measurable and adjustable restraining force until the cell is no longer able to move in a direction opposite to the applied force. Fukui et al. 1] reported on an experiment where amoeboid cells were imaged while they crawled against an artificial gravity field created by a centrifuge. An unexpected observation was that the net applied force on the amoeba did not seem to be the primary factor that limited its ability to climb. Instead, it appeared that the amoeba stalled when it was no longer able to support a pseudopodium against the applied gravity field. The high g-load bend the pseudopodium thereby preventing its attachment to the target point directly ahead of the cell. In this paper we further refine this idea by identifying the bending of the pseudopodium with the onset of elastic instability of a beam under its own weight. It is shown that the principal features of the experiment may be understood through this model and an estimate for the limiting g-load in reasonable accord with the experimental measurements is recovered.
\end{abstract}

Motility is a fundamental trait that distinguishes living things. The macroscopic motion of plants and animals can ultimately be reduced to motion on the level of single cells [2]. The movement of single cells also play a pivotal role in phenomena not directly related to movement of the whole organism. Thus, the morphogenetic migration of cells is responsible for the appearance of form and structure in embryogenesis. The movement of cells is crucial in the process of wound healing and in the functioning of the immune system and unfortunately in metastasis, where cancer cells spread from the primary tumor to invade other organs of the body [3]. When the environment of the cell is a fluid, the cell swims, but 
on a substrate or in a fibrous network, cells crawl. The mechanics of crawling [4, 5, 6, 7, 8, is a fundamental problem in biomechanics that has not yet been fully understood.

The measurement of the actual forces exerted by crawling cells on substrates is of obvious importance and various techniques have been employed in order to perform such measurements [9]. One method is to restrain the cell in some way, for example by holding a micro-needle in its path or by applying a suction force with a micro-pippette. An ingenious non-invasive technique involves imaging the wrinkles on an elastic substrate from which the applied force may be inferred [10]. Restraining forces can be applied to crawling cells by attaching magnetic beads to them and pulling with magnetic fields. Gravity is an excellent candidate for an external force as it does not require anything to be attached to the cell and it is the least likely to affect the natural behavior of the cell. Fukui et al. [1] reported on an experiment in which amoebae of Dictyostelium discoideum were allowed to crawl against an artificial gravity field created by a centrifuge. They determined the maximum g-force at which the amoeba "stalled" - that is, was unable to crawl in a direction opposite to the gravity field. However, a rather surprising finding was that the net force alone did not limit the crawling ability of the amoeba. Indeed, when the culture medium was replaced by one of a higher density, so that the amoeba was actually buoyant in it, it nevertheless stalled, even though, in this case, the gravity field was pushing the cell in the direction of motion. Fukui et al. observed that what appeared to limit the crawling ability of the amoeba was not the net force on the cell but rather the inability of the cell to extend a pseudopodium against the large gravitational field generated by the centrifuge; the pseudopodium was observed to bend and therefore not able to attach to a point on the substrate directly ahead of the amoeba (see Figure 1). In the present study, we show that this proposal of Fukui et al. that the buckling instability of the pseudopodium is the major factor limiting the ability of cells to crawl against high gravitational forces, is supported by a quantitative analysis based on mechanics. We further suggest that the ability to crawl against high gravitational forces is significantly improved in cells that are able to actively generate an internal turgor pressure in the pseudopodium by virtue of the contractile forces in the posterior part of the cell cortex [7].

If one assumes, based on these observations, that the structural stability of the actin rich pseudopodium against the gravity field limits the crawling ability of the cell, then it should be possible to use classical results on the stability of elastic structures under gravity to estimate the stalling acceleration $\left(g_{c}\right)$. The relevant result is the classical EulerBernoulli theory which predicts that an elastic beam of uniform cross-section buckles under a compressive force $(F)$ if this force exceeds a critical value given by

$$
F=\frac{\alpha E I}{L^{2}}
$$

where $E$ is the Young's modulus of the material, $I$ is the area moment of inertia of the cross-section, $L$ is its length and the value of the numerical constant $\alpha$ depends on the conditions at the ends of the beam; for a beam clamped at one end and free at the other, 


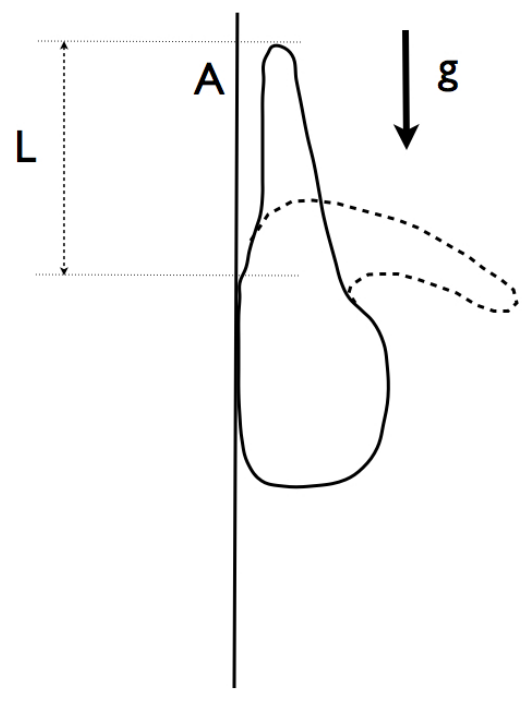

FiguRE 1. The amoeba stalls (is unable to move "up" against the artificial gravity field $g$ of the centrifuge) when the pseudopodium advancing in the direction of intended motion is bent by gravitational forces. Thus, the pseudopodium fails to attach at the target location $\mathrm{A}$ on the substrate directly above the cell body. Downward or lateral crawling of the cell is not prevented.

$\alpha=\pi^{2} / 4$. The Euler-Bernoulli theory proceeds from the assumptions of mechanical equilibrium and small deformations that result in a linear boundary value problem for the beam centerline. The requirement that this equation admit nonzero solutions results in an eigenvalue problem, and Eq. 1. corresponds to the lowest eigenvalue [11. If the compressive force is the weight of the beam $(W)$, a simple estimate for the maximum weight up to which the vertical configuration could be stable may be obtained by assuming all of the weight to be concentrated at the center of mass, so that

$$
W=\frac{4 \alpha E I}{L^{2}} .
$$

A more careful analysis where the weight is assumed to be uniformly distributed along the beam was provided by Greenhill [12]. Eq. 2 was found to hold except that $\alpha \approx 1.99$. For tapered beams Eq. 2 may still be applied if $I$ is regarded as the area moment of inertia of the base. Greenhill showed that for a right circular cone $\alpha \approx 2.54$ and for a paraboloid of revolution $\alpha \approx 2.47$. Keller and Niordson [13] calculated the greatest height $L$ that a beam of fixed weight $W$ can have if it is allowed to taper in an arbitrary way while preserving the shape of the cross-section. Once again, the result can be expressed in the form of Eq. 2 
with $\alpha \approx 8.23$. Thus, Eq. 2, where $\alpha$ is a numerical constant that has a value roughly between one and ten, may be used to estimate the critical acceleration $g_{c}$ of the centrifuge. For this purpose, one can rewrite Eq. 2 as

$$
g_{c}=\frac{4 \alpha E I}{(\Delta \rho) V L^{2}},
$$

using the apparent weight [16] (in place of $W$ ) of the pseudopodium which is the difference in density between the pseudopodium and the culture medium $(\Delta \rho)$ multiplied by the volume $(V)$ of the pseudopodium 1

If the pseudopodium is regarded as a circular cylinder of diameter $2 \mu \mathrm{m}$ and length $5 \mu \mathrm{m}$, then $I \approx 0.8 \times 10^{-24} \mathrm{~m}^{4}$ and $V \approx 1.6 \times 10^{-17} \mathrm{~m}^{3}$. Fukui et al [1] estimate that the density of the actin rich pseudopodium must be at least $\rho=1.124 \mathrm{gm} / \mathrm{cm}^{3}$. If we accept this value, then, since the medium density (at $0 \%$ Percoll) is $\rho_{0}=1.005 \mathrm{gm} / \mathrm{cm}^{3}, \Delta \rho=\rho-\rho_{0}=0.119$ $\mathrm{gm} / \mathrm{cm}^{3}$. The greatest uncertainty arises in estimating the Young's modulus $E$. If the pseudopodium is presumed to be supported predominantly by the mechanical strength of the actin network one could use in vitro measurements of the strength of actin gels. Such measurements are usually expressed in terms of the shear modulus $G$ which is related to $E$ and the bulk modulus $K_{V}$ as $E=9 G K_{V} /\left(3 K_{V}+G\right) \approx 3 G$ (since $G / K_{V}$ is smaller than $10^{-7}$ ). In vitro measurements show that $G$ for actin networks vary from about 300 $\mathrm{Pa}$ in the "gel" state to a value three orders of magnitude lower [14, 15] in the fluid state. The measured value depends primarily on the density and length of actin filaments and the density of cross-links created by various actin binding proteins (ABPs). If we take $G \approx 300 \mathrm{~Pa}$ and $\alpha \approx 2.5$ (corresponding to a structure of paraboloid shape) Eq. 3 yields a numerical estimate $g_{c} \sim 5 \times 10^{4} \mathrm{~m} / \mathrm{s}^{2}$.

In the experiment [1] Fukui et al. found that the myosin II knockout mutant (HS1) of the Dictyostelium amoeba stalled at $g_{c} \approx 3.9 \times 10^{4} \mathrm{~m} / \mathrm{s}^{2}$ (in the buffer without Percoll) which is in reasonable accord with the above estimate. The wild type strain (NC4) containing myosin II did not stall even at the highest accelerations tested (about $11.2 \times 10^{4} \mathrm{~m} / \mathrm{s}^{2}$ ). This is probably because the wild type cells are able to create considerable turgor pressure due to the myosin II-dependent contractile forces in the actin cortex thereby stiffening the pseudopodium. This case is discussed next.

In order to understand the effect of turgor pressure one needs to recognize that the pseudopodium is a poroelastic solid. When a beam made of such a material bends, the compression of the pores on one side of the neutral line results in a pressure rise in the interstitial fluid which then drives a flow across the beam. On account of viscous resistance, the fluid

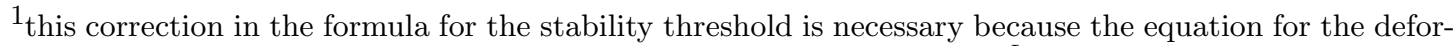
mation $y(x)$ of the beam is obtained by minimizing the energy functional $\int_{0}^{L} d x\left[E I\left(y^{\prime \prime}\right)^{2} / 2+\left(\rho-\rho_{0}\right) g \delta X\right]$ where $\delta X$ is the vertical displacement of a point on the beam at location ' $x$ ' due to beam curvature and $\rho_{0}$ is the density of the external medium. This differs from the corresponding expression in the absence of the external medium in that the density difference $\Delta \rho=\rho-\rho_{0}$ replaces the density of the material of the beam, $\rho$.
} 

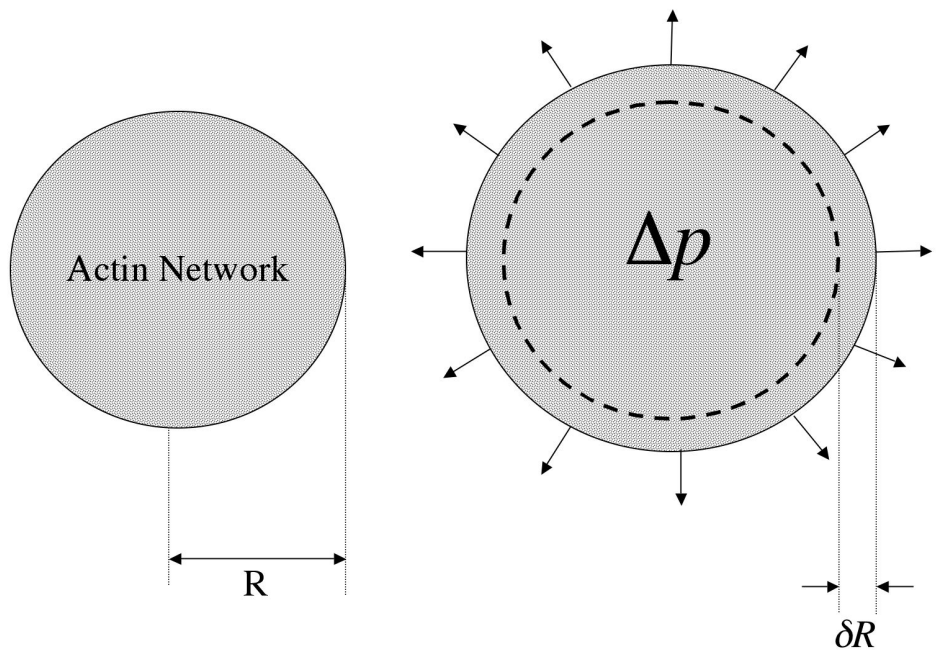

FIGURE 2. Diagram illustrating the radial expansion of the pseudopodium as a consequence of the rise in internal turgor pressure, resulting in a "stiffening" of the structure against elastic buckling.

responds to the bend with a time lag so that the system behaves much like a coupled massspring-damper system [17]. However, this mechanism does not alter the stability limit since the bifurcation at the onset of elastic instability takes place at zero frequency. Internal hydrostatic pressure stiffens the structure by one of two mechanisms (a) the swelling and consequent stretching of elastic elements may put it in a regime where the stress strain relation is no longer linear (b) the swelling may alter geometrical parameters (specifically, the parameter $I$ in Eq. 3). The first of these effects is less likely, though a careful estimate is difficult as not much information is available on the nonlinear elasticity of actin gels. However, the second of these effects is readily estimated. The situation is depicted in Figure 2. Since an element of the cell membrane is in equilibrium due to the balance of an outward pressure $(\Delta p)$ and an inward elastic stress $E(\delta R / R)$ where $\delta R$ is the increase in the radius $R$, we have $\delta R / R=(\Delta p) / E$. Thus,

$$
1+\frac{\delta g_{c}}{g_{c}}=\left(1+\frac{\delta R}{R}\right)^{4}=\left(1+\frac{\Delta p}{E}\right)^{4}
$$

since the area moment of inertia of a cylinder is $I=\pi R^{4} / 4$. In the experiment [1] the wild type strain (NC4) containing myosin II did not stall at the maximum acceleration of $g_{c}=11.2 \times 10^{4} \mathrm{~m} / \mathrm{s}^{2}$, suggesting that $\delta g_{c} / g_{c}>1.9$. Thus, $\Delta p>0.305 \times E=275 \mathrm{~Pa}$, using the value $E=3 G=900 \mathrm{~Pa}$ cited earlier. Pasternak et al. [18 report a difference 
$\Delta T \approx 0.13$ mdyn per micron in the cortical tension between the strains AX4 and the myosin lacking mhcA- strain of Dictyostelium both in the resting phase. If one converts this number to a pressure using the Laplace formula for surface tension, one obtains the estimate for the myosin generated pressure: $\Delta p=2 \Delta T / R \sim 520$ Pa where $R \sim 5 \mu \mathrm{m}$ is taken as a characteristic radius of the cell. Thus, our rough estimate $\Delta p>275 \mathrm{~Pa}$ is not inconsistent with reported values for myosin II-dependent pressures in the cell cortex that may be inferred on the basis of existing experimental data.

In conclusion, the hypothesis advanced by Fukui et al. that the ability of the Dictyostelium amoeba to support a pseudopodium against a strong gravity field limits its ability to crawl against such a field appear to be supported by the present analysis based on the mechanics of the buckling of elastic structures. Similar ideas have long been used in the field of plant biomechanics [19], but the centrifuge experiments of Fukui et al. present an opportunity for an application of these concepts to the mechanics of cell crawling. In a broader context, we illustrate that the interpretation of experiments in which one attempts to quantify the force applied by a crawling cell on a substrate by measuring an applied restraining force [9] may be subtle, because, the limiting factor may not be the ability of the cell to pull against the applied force but rather a failure in some other aspect of the motility mechanism of the cell (e.g. contact inhibition [20]).

Acknowledgements: We would like to thank Howard A. Stone and Joseph B. Keller for reading and commenting on a draft of the manuscript and L. Mahadevan for helpful discussions relating to the bending of poroelastic beams.

\section{REFERENCES}

[1] Y. Fukui, T. Q. P. Uyeda, C. Kitayama, and S. Inoué, "How well can an amoeba climb?," Proceedings of the National Academy of Sciences of the United States of America 97, 10020-10025 (2000).

[2] D. Bray, Cell Movements, first trade paperback ed. (Garland Science, 1992).

[3] S. Li, J. L. Guan, and S. Chien, "Biochemistry and biomechanics of cell motility," Annual Review of Biomedical Engineering 7, 105-150 (2005).

[4] D. A. Fletcher and J. A. Theriot, "An introduction to cell motility for the physical scientist," Physical Biology 1, T1-T10 (2004).

[5] T. P. Stossel, "On the crawling of animal cells," Science 260, 1086-1094 (1993).

[6] T. P. Stossel, "The machinery of cell crawling," Scientific American 271, 54-55, 58-63 (1994), PMID: 8091190.

[7] Y. Fukui, "Toward a New Concept of Cell Motility: Cytoskeletal Dynamics in Amoeboid Movement and Cell Division," in International Review of Cytology, K. W. Jeon and J. Jarvik, eds., (Academic Press, 1993), Vol. 144, pp. 85-127.

[8] J.B. Keller and M.S. Falkovitz, "Crawling of worms," Journal of Theoretical Biology 104, 417-442 (1983).

[9] T. Oliver, J. Lee, and K. Jacobson, "Forces exerted by locomoting cells," Seminars in Cell Biology 5, 139-147 (1994), PMID: 7919227.

[10] M. Dembo and Y. Wang, "Stresses at the Cell-to-Substrate Interface during Locomotion of Fibroblasts," Biophysical Journal 76, 2307-2316 (1999). 
[11] S. P. Timoshenko and J. M. Gere, Theory of Elastic Stability, 2 ed. (Dover, Mineola, New York, 2009).

[12] A. Greenhill, "Determination of the greatest height consistent with stability that a vertical pole or mast can be made, and of the greatest height to which a tree of given proportions can grow," Proceedings of the Cambridge Philosophical Society 4, 65-73 (1881).

[13] J. B. Keller and F. I. Niordson, "The tallest column," Journal of Mathematics and Mechanics 16, 433-446 (1966).

[14] P. A. Janmey, S. Hvidt, J. Käs, D. Lerche, A. Maggs, E. Sackmann, M. Schliwa, and T. P. Stossel, "The mechanical properties of actin gels. Elastic modulus and filament motions," The Journal of Biological Chemistry 269, 32503-32513 (1994), PMID: 7798252.

[15] M. L. Gardel, J. H. Shin, F. C. MacKintosh, L. Mahadevan, P. Matsudaira, and D. A. Weitz, "Elastic behavior of cross-linked and bundled actin networks," Science (New York, N.Y.) 304, 1301-1305 (2004), PMID: 15166374 .

[16] T. Kokkinis and M. M. Bernitsas, "Effect of fluid static pressure on the immediate postbuckling behavior of heavy tubular columns," Applied Ocean Research 9, 141-149 (1987).

[17] J. M. Skotheim and L. Mahadevan, "Dynamics of poroelastic filaments," Proceedings of the Royal Society of London (A), 460, 1995-2020 (2004).

[18] C. Pasternak, J. A. Spudich, and E. L. Elson, "Capping of surface receptors and concomitant cortical tension are generated by conventional myosin," Nature 341, 549-551 (1989).

[19] S. Vogel, "Living in a physical world VII. Gravity and life on the ground," Journal of Biosciences $\mathbf{3 1}$, 201-214 (2006).

[20] P. Weiss, "Guiding principles in cell locomotion and cell aggregation," Experimental Cell Research 8, 260-281 (1961). 